\title{
Quantification and Control of Non-Markovian Evolution in Finite Quantum Systems via Feedback
}

\author{
N. Chancellor, ${ }^{1,2}$ C. Petri, ${ }^{1}$ L. Campos Venuti, ${ }^{1}$ A.F.J. Levi, ${ }^{3,1}$ and S. Haas ${ }^{1,4}$ \\ ${ }^{I}$ Department of Physics and Astronomy and Center for Quantum Information Science \& Technology, \\ University of Southern California, Los Angeles, CA 90089-0484, USA \\ ${ }^{2}$ London Centre for Nanotechnology, University College London, London WC1H OAH, UK \\ ${ }^{3}$ Department of Electrical Engineering, University of Southern California, Los Angeles, CA 90089-2533, USA \\ ${ }^{4}$ School of Engineering and Science, Jacobs University Bremen, Bremen 28759, Germany
}

\begin{abstract}
We consider the unitary time evolution of continuous quantum mechanical systems confined to a cavity in contact with a finite bath of variable size. We define a new measure relating to (non-)Markovianity which parallels the standard one for the case of integrable Lindbladian dynamics but has the advantage of being numerically tractable also for large many particles systems. The relevant time scales are identified, which characterize nonMarkovian transient behavior, boundary scattering induced non-Markovian oscillations at intermediate times, and non-Markovian rephasing events at long time scales. It is shown how these time scales can be controlled by tunable parameters such as the bath size and the strength of the system-bath coupling.
\end{abstract}

\section{INTRODUCTION}

It is well known that the time evolution of quantum closed systems at finite size is intrinsically non-Markovian. The reason is that the reduced density matrix is necessarily an oscillating function which admits no infinite-time limit. In the quantum case, the time scale for partial rephasing events (revivals) is proportional to the system size, whereas full rephasing (Poincare recurrences) can occur at an astronomically large time scales [1, 2]. Nonetheless, previous studies of the transient dynamics in lattice models have revealed extended time domains within which the time evolution is pseudoMarkovian in the sense that memory of the initial state appears to be lost [3]. Strictly speaking, this conclusion would of course be erroneous, as this information is only temporarily dispersed among the system's accessible modes before it reemerges during rephasing events. However, this observation raises the interesting question if and how the time scales which determine such pseudo-Markovian time evolution (i.e. Markovian when restricted to a definite time domain) as well as nonMarkovian features can be controlled.

Here we consider the illustrative example of a continuous one-dimensional array with periodic boundary conditions. We divide this array into an active "system" in region $A$ connected to a region $B$ which acts as a bath. A pair of thin barriers are than placed between the system and the bath. The concept of a finite bath is different from the conventional notion of an infinite reservoir, but this setup allows us to explore the size of the bath as a non-trivial tuning parameter, along with the system-bath coupling. In previous work, the non-Markovian dynamics in such arrangements have been explored using lattice Hamiltonians [3]. It was found that by breaking certain symmetries, features signaling non-Markovian time evolution disappear within a finite time scale which is governed by the system-bath coupling as well as by the amount of random symmetry breaking introduced in the bath Hamiltonian. Here, randomness raises the number of accessible states of the bath.

In this paper, we investigate control over the time evolution of continuum Hamiltonians, tuning their bath degrees of freedom by adjusting the bath size relative to the system. While this study is kept at a relatively abstract level, primarily focus-

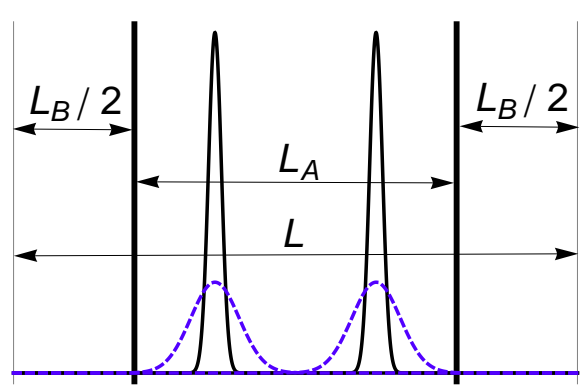

Figure 1: (Color online) Illustration of the finite system-bath array considered here. The system-bath boundaries are shown as thick vertical lines, along with two sets of initial Gaussian wave functions: broad (dashed line) and narrow (continuous line). Except otherwise stated, delta barriers on the left system-bath boundary are of strength $V_{1}=10^{6}$ and on the right boundary are of strength $V_{2}=2.0 \times 10^{6}$. The bath segments are connected via periodic boundary conditions.

ing on the emerging time scales, experimental realizations of such configurations can easily be imagined in the context of coupled laser cavities, connected by semi-transparent mirrors, or in the context of electron wave packets tunneling through barriers in layered $(\mathrm{Ga}, \mathrm{Al}) \mathrm{As}$ heterostructures. In order to avoid the pitfalls of a more conventional treatment of open quantum systems based on the Lindblad formalism [4], including infinitesimal system-bath coupling and forced Markovianity, we study the time evolution of a complete, untruncated system-bath Hamiltonian. The price we have to pay for this is the restriction to non-interacting systems described by effective single-particle Hamiltonians, in order to keep the problem numerically manageable.

We will first introduce the concept of free Markovianity (FM) which parallels the definition of Markovianity ala Breuer-Laine-Piilo (BLP) [5], for the case in which the Lindblad, Markovian dynamics, is integrable in the sense of [6]. Free Markovianity differs from BLP Markovianity in the general case, however the two definitions become equivalent when restricted to integrable dynamics. The resulting measure of free non-Markovianity (FNM), $D(t)$, detects a free non-Markovian event whenever $D(t)$ is an increasing function of time, in analogy with the BLP measure [5]. It should 
be reminded at this point that properly quantifying the amount of non-Markovianity of a given dynamics has been subject of intense research in recent times and many different, nonnecessarily equivalent, measures of non-Markovianity have been proposed [5, 7-11]. The measure $D(t)$ that we propose, although identifies yet another class of non-Markovian systems, has a series of advantages with respect to the measures that have appeared so far. The main point being that $D(t)$ can easily be computed even for many-body systems composed of many particles [21]. Moreover the quantity $D(t)$ is expressed in terms of single particles quantities thus allowing for a simple physical interpretation. While in general, the maximum of $D(t)$ over all possible initial states has to be calculated, even a single pair of linearly independent states can provide valuable insight into the overall dynamics of the system. We will refer to the distance between such a pair as $D_{\Gamma_{1}, \Gamma_{2}}(t)$, where $\Gamma$ are the covariance matrix of the two states.

This paper is organized as follows. In the next section we define our measure of free non-Markovianity for noninteracting systems. We then describe the general framework. This is followed by an analysis of the characteristic timescales that can be extracted from the numerics. We conclude with a discussion of how feedback via tunable bath parameters can be utilized to control the time evolution in physically relevant systems.

\section{MEASURE OF FREE NON-MARKOVIANITY FOR NON-INTERACTING SYSTEMS}

We proceed here to define a measure of free nonMarkovianity for a system of non-interacting particles. The results apply both to Fermi-Dirac and Bose-Einstein statistics although with some technical caveats in the latter case. The proper definition of a measure of non-Markovianity is an issue of current debate [5, 8, 11-13]. For our purposes it seems appropriate to take the point of view of Breuer-Laine-Piilo (BLP) [5] which provides a physical interpretation in terms of information flow. According to [5] a violation of Markovianity is detected whenever the quantity $\left\|\rho_{1}(t)-\rho_{2}(t)\right\|_{1}$ increases in time. Here $\|\cdot\|_{1}$ denotes the trace norm and $\rho_{i}(t)$ are the density matrices of the system of interest at time $t$, corresponding to different initial conditions $\rho_{1}(0), \rho_{2}(0)$. As it turns out the trace norm $\left\|\rho_{1}(t)-\rho_{2}(t)\right\|_{1}$ is difficult to compute even for the simplest case of systems composed of a single qubit [5]. Therefore we take a different approach.

Consider a many-body system whose dynamical evolution is not necessarily unitary, but it is assumed to be noninteracting in a sense that we are going to specify shortly. Suppose that the quantum process is described by a Markovian master equation,

$$
\frac{d \rho}{d t}=\mathcal{L} \rho
$$

with generator $\mathcal{L}$ in Lindblad form

$$
\mathcal{L} \rho=-i[H, \rho]+\sum_{i} \gamma_{i}\left[A_{i} \rho A_{i}^{\dagger}-\frac{1}{2}\left\{A_{i}^{\dagger} A_{i}, \rho\right\}\right] .
$$

In the spirit of [6] we call the dynamics non-interacting if the Hamiltonian $H$ is quadratic in the canonical creation and annihilation operators whereas the Lindblad terms $A_{i}$ are linear combinations thereof. The solution of such a free Markovian master equations is conveniently encoded in terms of the covariance matrix or two-point correlation function $\Gamma_{j \alpha, k \beta}$ [14-16]. For Fermions the covariance matrix has the form $\Gamma_{j \alpha, k \beta}=-\operatorname{Im}\left[\operatorname{tr}\left(\rho \omega_{j}^{\alpha} \omega_{k}^{\beta}\right)\right]$ where the the Majorana operators $\omega_{j}^{\alpha}$ are given by $\omega_{j}^{1}=f_{j}+f_{j}^{\dagger}, \omega_{j}^{2}=i\left(f_{j}-f_{j}^{\dagger}\right)$ in terms of Fermi operators $f_{i}$. For bosons instead one has $\Gamma_{j \alpha, k \beta}=$ $\operatorname{Re}\left[\operatorname{tr}\left(\rho u_{j}^{\alpha} u_{k}^{\beta}\right)\right]$ for quadrature operators $u_{j}^{1}=b_{j}+b_{j}^{\dagger}$ and $u_{j}^{2}=i\left(b_{j}-b_{j}^{\dagger}\right)$ and canonical Bose operators $b_{j}$ (see Ref. [16] for more details).

From Equation (1) one finds that the covariance matrix $\Gamma$ satisfies the equation of motion [16]

$$
\frac{d \Gamma}{d t}=X^{T} \Gamma+\Gamma X-Y,
$$

with matrices $X, Y$ which depend on $H$ and $A_{i}$. In the Fermionic case one can show that the spectrum of $X$ lies in the sector $\operatorname{Re}(z) \geq 0$ of the complex plane [14, 16]. This in turns implies that the dynamics given by Eq. (1) gives rise to a contractive flow. In other words, using following basis-dependent identification between matrices and vectors ||$n\rangle\langle m|\gg:=| n, m\rangle$ (see e.g. [17] Sec. 2.4) given two different initial conditions $\Gamma_{1}(0), \Gamma_{2}(0)$, one has 22]

$$
\left\|\left|\Gamma_{1}(t) \gg-\right| \Gamma_{2}(t) \gg\right\| \leq\left\||| \Gamma_{1}(s) \gg-\mid \Gamma_{2}(s) \gg\right\| \text {, for } s \leq t .
$$

The same result does not apply directly in the case of bosons essentially because the mapping $\rho \rightarrow \Gamma[\rho]$ is not continuous. Physically this corresponds to the possibility of pumping-in infinite energy in the bosonic fields [14, 16]. A standard procedure to avoid such infinities is to introduce a (very large) cut-off in the number of particle thus avoiding infinite energy states. With this prescription Eq. (4) holds also for bosons.

The norm appearing in Eq. (4) is in principle any norm for the vectors $\mid \Gamma_{j}(t) \gg$. The $\ell^{2}$ norm seems to be the most natural one which induces a basis-independent norm on the matrices $\Gamma_{j}(t)$. The induced norm in this case is the Hilbert-Schmidt (HS) norm for the matrices $\Gamma_{j}$, and one has $\|X \gg\|_{\ell^{2}}=\|X\|_{H S}=\sqrt{\operatorname{tr}\left(X^{\dagger} X\right)}$.

To summarize, the BLP measure of Markovianity [5] is defined as the trace distance between two density matrices,

$$
D_{\rho_{1}, \rho_{2}}^{B L P}(t)=\left\|\rho_{1}(t)-\rho_{2}(t)\right\|_{1}
$$

while our measure of free Markovianity is defined as the Hilbert-Schmidt distance between two covariance matrices

$$
D_{\Gamma_{1}, \Gamma_{2}}(t)=\left\|\Gamma_{1}(t)-\Gamma_{2}(t)\right\|_{H S} .
$$

In the spirit of Ref. [5] we now single out a violation of free Markovianity if, for some initial states $\Gamma_{1}(0), \Gamma_{2}(0)$, the norm $\left\|\Gamma_{1}(t)-\Gamma_{2}(t)\right\|_{H S}$ is not decreasing. In other words, calling $\sigma\left(t, \Gamma_{1}, \Gamma_{2}\right):=(d / d t) D_{\Gamma_{1}, \Gamma_{2}}(t)=$ 
$(d / d t)\left(\left\|\Gamma_{1}(t)-\Gamma_{2}(t)\right\|_{H S} / 2\right)$, we say that the dynamics is free Markovian (FM) if for some time interval and initial states with covariance matrix $\Gamma_{1,2}(0)$, we have $\sigma\left(t, \Gamma_{1}, \Gamma_{2}\right) \leq$ 0 . In principle one could even define a measure of free nonMarkovianity paralleling the definition of [5] for quasi-free dynamics. The quantity:

$$
\mathcal{N}=\sup _{\Gamma_{1}, \Gamma_{2}} \int_{\sigma>0} \sigma\left(t, \Gamma_{1}, \Gamma_{2}\right) d t,
$$

encodes the amount of free non-Markovianity in the process dynamics from $t=0$ to $t=\infty$. As we will see, for oneparticle states $\left\|\Gamma_{1}(t)-\Gamma_{2}(t)\right\|_{H S}$ has a particularly simple form so that one can hope to be able to perform the maximization in Eq. (7). This project is left for future investigations.

In general free Markovianity is different from the notion of Markovianity a-la BLP. However, when restricted to the class of free, Lindblad dynamics the two definitions become equivalent.

Our procedure will be the following. We will consider a system of identical particles described by a free Hamiltonian dynamics. The setting is relevant to electrons, so that particles will be fermions, but, as shown above, the statistic is essentially unimportant modulo a technical caveat. Restricting to free Hamiltonians will allow us to easily integrate the equation of motions going to the one particle sector. We then consider the sub-dynamics of the system, obtained by tracing over the bath degrees of freedom. The central question we ask, is if such sub-dynamics can be described by a freeMarkovian master equation. To this end we compute the quantity in Eq. (4) for different initial states and Hamiltonian parameters. Note that the result, Eq. (4), is expressed in terms of single-particle quantities. This fact will make the physical interpretation simpler. In the following, for simplicity of language, we will simply refer to free (non) Markovianity as (non) Markovianity. The definition given here is intended throughout unless otherwise specified.

\section{METHODS}

We now explain the general setting. We place a single particle in a one-dimensional array of length $L=L_{A}+L_{B}$, with periodic boundary conditions (PBC), i.e. a ring. We will later trace out the segment $B$ of length $L_{B}$ which plays the role of external bath, whereas $A$ is the system of interest. The oneparticle Hamiltonian is of the form $H=-\partial^{2} / \partial x^{2}+V(x)$ where the external potential $V(x)$ will be specified later. In a particle-number conserving system the covariance matrix $\Gamma$ is a function of $R_{x, y}:=\operatorname{tr}\left(\rho c_{x}^{\dagger} c_{y}\right)$ only and one has $\|\Gamma\|_{H S}=\sqrt{8}\|R\|_{H S}$ [23]. The system is initialized in the one-particle state $|\psi(0)\rangle$, which is then evolved according to $|\psi(t)\rangle=e^{-i t H}|\psi(0)\rangle$. In the Fock space this corresponds to the state $|\Psi(t)\rangle=c_{\psi(t)}^{\dagger}|0\rangle$ where $c_{f}^{\dagger}$ create a Fermion or Boson in state $f$. Now, in the position "basis" $|x\rangle,|\psi(t)\rangle=\sum_{x} \psi(x, t)|x\rangle$ and $c_{\psi(t)}^{\dagger}=\sum_{x} \overline{\psi(x, t)} c_{x}^{\dagger}$ and one obtains the following integral kernel

$$
R_{x, y}=\overline{\psi(x, t)} \psi(y, t) .
$$

The full density matrix is a many body state $\rho(t)=$ $|\Psi(t)\rangle\langle\Psi(t)|$, and tracing out the bath $B$ corresponds to discarding from $R$ in Eq. (8) all the labels $(x, y)$ belonging to $B$, i.e. projecting $R$ onto $A$. The one-particle Hilbert space is decomposed into a direct sum $\mathcal{H}=\mathcal{H}_{A} \oplus \mathcal{H}_{B}$, where $\mathcal{H}_{A / B}$ describes wave functions with support only in $A / B$. Calling $P_{A}$ the operator which projects onto $\mathcal{H}_{A}$, the restriction of $R=|\psi\rangle\langle\psi|$ on $\mathcal{H}_{A}$ is $R^{A}=P_{A}|\psi\rangle\left\langle\psi\left|P_{A}=\right| \psi_{A}\right\rangle\left\langle\psi_{A}\right|$, for a non-normalized state $\left|\psi_{A}\right\rangle$. With the notation $\rho_{R}$ for the Gaussian state given by covariance $R$, the tracing out the bath is simply achieved via $\operatorname{tr}_{B} \rho_{R}=\rho_{R^{A}}$. In the general case in which the initial states has $N$ particles, $R$ and $R^{A}$ are rank $N$ operators. Considering the evolution of two different initial states $\left|\psi_{j}(0)\right\rangle$, the difference of covariance matrices restricted to region $A$ is

$$
P_{A}\left(\left|\psi_{1}\right\rangle\left\langle\psi_{1}|-| \psi_{2}\right\rangle\left\langle\psi_{2}\right|\right) P_{A}=\left|\psi_{1, A}\right\rangle\left\langle\psi_{1, A}|-| \psi_{2, A}\right\rangle\left\langle\psi_{2, A}\right|
$$

with unnormalized states $\left|\psi_{j, A}\right\rangle$ supported in $\mathcal{H}_{A}$. In the, nonorthonormal, basis $\left|\psi_{j, A}\right\rangle$ the above operator has the form

$$
\left(\begin{array}{cc}
p_{1,1} & p_{1,2} \\
-\overline{p_{1,2}} & -p_{2,2}
\end{array}\right)
$$

where

$$
\begin{aligned}
p_{i, j} & =\left\langle\psi_{i, A} \mid \psi_{j, A}\right\rangle \\
& =\left\langle\psi_{i}(t)\left|P_{A}\right| \psi_{j}(t)\right\rangle \\
& =\sum_{k, q} e^{i t\left(E_{k}-E_{q}\right)}\left\langle\psi_{i} \mid \phi_{k}\right\rangle\left\langle\phi_{q} \mid \psi_{j}\right\rangle \Delta_{L}^{A}(k, q)
\end{aligned}
$$

with $i, j=1,2$ and having defined

$$
\Delta_{L}^{A}(k, q)=\left\langle\phi_{k}\left|P_{A}\right| \phi_{q}\right\rangle=\int_{A} \overline{\phi_{k}(x)} \phi_{q}(x) d x .
$$

The term $p_{j, j}$ gives the probability that the particle initialized in $\left|\psi_{j}\right\rangle$ is in region $A$ at time $t$. Our indicators of nonMarkovianity are given in terms of the eigenvalues of the matrix (10) which read

$$
\lambda_{1,2}=\frac{\left(p_{1,1}-p_{2,2}\right) \pm \sqrt{\left(p_{1,1}+p_{2,2}\right)^{2}-4\left|p_{1,2}\right|^{2}}}{2} .
$$

Finally, according to the discussion in Sec. II the distance that we consider to characterize non-Markovianity is given by

$$
\begin{aligned}
D_{\psi_{1}, \psi_{2}} & =\frac{1}{\sqrt{2}}\left\|R_{1}^{A}(t)-R_{2}^{A}(t)\right\|=\frac{1}{\sqrt{2}} \sqrt{\lambda_{1}^{2}+\lambda_{2}^{2}} \\
& =\frac{1}{\sqrt{2}} \sqrt{p_{1,1}^{2}+p_{2,2}^{2}-2\left|p_{1,2}\right|^{2}},
\end{aligned}
$$

where $R_{j}^{A}(t)$ are the covariance matrices of system $A$ at time $t$. A factor $1 / \sqrt{2}$ has been inserted to scale the measure, so that its maximum value is 1 (attained when $p_{1,1}=p_{2,2}=$ 1 and $p_{1,2}=0$ ). Note that by Schwartz inequality one has $\left|p_{1,2}\right|^{2} \leq p_{1,1} p_{2,2}$ implying that $D_{\psi_{1}, \psi_{2}}$ is indeed real. 
The final result Eq. (17) is extremely simple and physically quite compelling. Assume for simplicity that the states are orthogonal on $A$ (so that $p_{1,2}=0$ ). Eq. (17) then is simply the geometric mean of the probabilities of the particles being in region $A$. As such, as a function of $t$, it is quite clear that $D_{\psi_{1}, \psi_{2}}(t)$ increases when particles move into region $A$ signaling a violation of Markovianity. Since $D_{\psi_{1}, \psi_{2}}(t)$ is the (Hilbert-Schmidt) distance of two covariance matrices it trivially characterizes the distinguishability of the $R_{j}^{A}(t)$. The "information flow" of the BLP measure becomes in this setting a flow of probability. With slight abuse of language we will speak of Markovian behavior when $D_{\psi_{1}, \psi_{2}}(t)$ decreases in time although this is only consistent with Markovian dynamics. This is in accordance with the general intuition of Markovian evolution occurring due to information leakage from the system.

\section{RESULTS}

We consider two specific initial Gaussian packets localized around $x_{j}: \psi_{j}(x)=C_{j} e^{-\left(x-x_{j}\right)^{2} /\left(4 \sigma_{j}^{2}\right)}$ $(j=1,2)$. The normalization factor is given by the equation $\quad C_{j}^{2} \sqrt{\pi / 2} \sigma_{j}\left\{\operatorname{Erf}\left[\left(L-2 x_{j}\right) /\left(\sqrt{8} \sigma_{j}\right)\right]\right.$ $\left.+\operatorname{Erf}\left[\left(L+2 x_{j}\right) /\left(\sqrt{8} \sigma_{j}\right)\right]\right\}=1$, [24] which boils down to $C_{j} \simeq\left(2 \pi \sigma_{j}^{2}\right)^{-1 / 4}$ for $\sigma_{j} \ll L$. We choose initial states symmetrically displaced with respect to the origin, i.e. $x_{1}=-x_{2}$ as shown in Fig. 1 In this case, for symmetric potentials $V(x)$ one has $p_{1,1}(t)=p_{2,2}(t)$ [25]. For such symmetric configurations $\lambda_{1}=-\lambda_{2}$, and one has the further simplification $D_{\psi_{1}, \psi_{2}}=\left|\lambda_{1}\right|=\left|\lambda_{2}\right|$. As a first example we consider in some detail the purely kinetic evolution corresponding to $V(x)=0$.

\section{A. Purely kinetic evolution}

Let us first consider the case when there are no delta barriers separating the system from the bath, as shown in the insets (a) and (c) of Fig. 3. The spectrum acquires the familiar form $E_{k}=k^{2}$ where the quasi-momenta satisfy $k=2 \pi n / L, n \in$ $\mathbb{Z}$. The "geometric" factor $\Delta_{L}^{A}$ becomes

$$
\Delta_{L}^{A}(k, q)=\frac{1}{L} \int_{L_{A}} e^{i x(k-q)} d x=\frac{\sin \left[L_{A}(k-q) / 2\right]}{[L(k-q) / 2]} .
$$

In this case we have simple dispersing wave packets. However, since the system-bath configuration has finite spatial extent, there are rephasing events (or revivals) that occur at time scales of the order of the total system size, i.e. $\tau_{L} \propto L$ [18, 19]. This timescale measures the time it takes for a packet to go around the periodic boundaries and come back. As Fig. 2 shows, recurrences are not present if the bath is infinite (discussed in more detail later). However, for a finite bath recurrences are observed in Fig. 3 (a) and (c), where the time evolution of the non-Markovian indicator between two initial wave packets is shown. In addition to these rephasing events one also observes smaller amplitude, more rapid oscillations in the non-Markovian indicator. A measure of these oscillations is roughly given by the effective numbers of Hamiltonian eigenstates needed to express the initial wave-packet. One can obtain this number by imposing that the fidelity of the initial state be $F=1-\epsilon$. One obtains that roughly $N^{*}=-(L / \sigma) \sqrt{-\ln (\epsilon)}$ eigenstates are required to obtain the desired fidelity. The number $N^{*}$ also gives the number of effective energy eigenstates involved in the dynamics. It is evident from the figure that broad initial wave-packets (Fig. 3 (c) and (d)) contain many more frequencies than narrow initial wave-packets (Fig. 3 (a) and (b)). In fact smaller $\sigma$, means larger $N^{*}$ so that the measure $D_{\psi_{1}, \psi_{2}}(t)$ contains more frequencies and consequently shows faster oscillations.

We conclude that for this most simple example nonMarkovian time evolution, i.e. deviations from a monotonically decaying HS distance, occurs at two time scales, $\tau_{L}$ controlled by the total system size, and $\tau_{\sigma}$ controlled by the width of the initial wave packets and their subsequent dispersion.

We consider now the situation where $L \rightarrow \infty$. In this case many time scales which depend on $L$, such as $\tau_{L}$, are sent to infinity. We then keep $L_{A}$ constant and send $L \rightarrow \infty$ in Eq. (13). In this limit $L^{-1} \sum_{k} \rightarrow(2 \pi)^{-1} \int_{\mathbb{R}} d k$, and we obtain

$p_{i, j}=\int_{\mathbb{R}} \frac{d k}{2 \pi} \int_{\mathbb{R}} \frac{d q}{2 \pi} e^{i t\left(k^{2}-q^{2}\right)} \overline{\hat{\psi}_{i}(k)} \hat{\psi}_{j}(q) \frac{\sin \left[L_{A}(k-q) / 2\right]}{[(k-q) / 2]}$

where the initial wave functions in Fourier space are given by

$$
\hat{\psi}_{j}(q)=\int_{\mathbb{R}} d x e^{i q x} \psi_{j}(x)=\sqrt{4 \pi \sigma_{j}^{2}} C_{j} e^{i q x_{j}} e^{-q^{2} \sigma_{j}^{2}}
$$

Changing variables to $r=(k-q), R=(k+q) / 2$, the integral over $R$ is Gaussian, and we obtain

$$
\begin{aligned}
p_{i, j} & =\int_{\mathbb{R}} d r \frac{\sin \left(r L_{A} / 2\right)}{\pi r} \exp \left\{-\frac{r^{2} \sigma_{t}^{2}}{2}\right. \\
& \left.+\frac{r}{2}\left[\frac{t}{\sigma^{2}}\left(x_{i}-x_{j}\right)-i\left(x_{i}+x_{j}\right)\right]-\frac{\left(x_{i}-x_{j}\right)^{2}}{8 \sigma^{2}}\right\}
\end{aligned}
$$

with $\sigma_{t}=\sqrt{\left(t^{2} / \sigma^{2}+\sigma^{2}\right)}$. Writing $\sin \left(L_{A} r\right) / r=$ $\int_{0}^{L_{A}} \cos (y r) d y$, the integral over $r$ is Gaussian, and one is left with an incomplete Gaussian integral over $y$. The final result is

$$
\begin{aligned}
p_{i, j}=\frac{e^{-\left(x_{i}-x_{j}\right)^{2} /\left(8 \sigma^{2}\right)}}{2} \times & \\
& {\left[\operatorname{Erf}\left(\frac{L_{A}^{+} \sigma^{2}+i t\left(x_{i}-x_{j}\right)}{\sqrt{8} \sigma_{t} \sigma^{2}}\right)\right.} \\
& \left.\quad+\operatorname{Erf}\left(\frac{L_{A}^{-} \sigma^{2}-i t\left(x_{i}-x_{j}\right)}{\sqrt{8} \sigma_{t} \sigma^{2}}\right)\right]
\end{aligned}
$$

having defined $L_{A}^{ \pm}=L_{A} \pm\left(x_{i}+x_{j}\right)$. Plugging Eq. (23) into 
Eq. (17) one obtains

$$
\begin{aligned}
\left(D_{\psi_{1}, \psi_{2}}\right)^{2}= & \frac{1}{8} \sum_{j=1}^{2}\left[\operatorname{Erf}\left(\frac{L_{A}-2 x_{j}}{\sqrt{8} \sigma_{t}}\right)+\operatorname{Erf}\left(\frac{L_{A}+2 x_{j}}{\sqrt{8} \sigma_{t}}\right)\right]^{2} \\
- & \frac{1}{4} e^{-\frac{\left(x_{1}-x_{2}\right)^{2}}{2 \sigma^{2}}} \mid \operatorname{Erf}\left(\frac{L_{A}^{+}}{\sqrt{8} \sigma_{t}}+i t \frac{\left(x_{1}-x_{2}\right)}{\sqrt{8} \sigma_{t} \sigma^{2}}\right) \\
& +\left.\operatorname{Erf}\left(\frac{L_{A}^{-}}{\sqrt{8} \sigma_{t}}-i t \frac{\left(x_{1}-x_{2}\right)}{\sqrt{8} \sigma_{t} \sigma^{2}}\right)\right|^{2}
\end{aligned}
$$
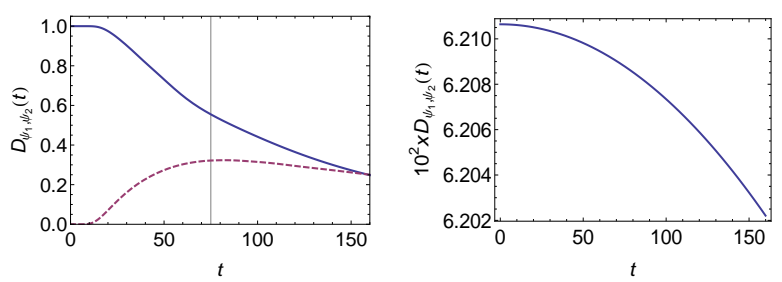

(24)Figure 2: (Color online) Markovian/non-Markovian character for an infinite bath. Left panel: continuous curve for initial states "inside region $A "\left(x_{1}=-x_{2}=10, L_{A}=60 \sigma=2\right)$, dashed curve, initial states outside region $A\left(x_{1}=-x_{2}=45, L_{A}=60 \sigma=2\right)$. Here a non-Markovian window appears for $0 \leq t \leq \tau_{M}$ with $\tau_{M} \approx$ $\sigma\left(x_{1}+L_{A} / 2\right) / 2$ indicated by the vertical line. Note that $D_{\psi_{1}, \psi_{2}}(t)$ starts decreasing roughly for $t \geq \tau_{M}$. Right panel: if the initial width $\sigma$ is cranked beyond a value proportional to $\left(x_{1}+L_{A} / 2\right) / 2$ the nonMarkovian window disappears. Here $x_{1}=-x_{2}=45, L_{A}=60$ $\sigma=60$. Note the very small vertical scale, i.e. $D_{\psi_{1}, \psi_{2}}(t)$ is almost constant.

we always expect Markovianity for any parameter value. Indeed this intuition can be confirmed after a lengthy calculation taking the time derivative of Eq. (24).

Let us now consider the other situation where the particles are initialized outside $A$, i.e. $\left|x_{i}\right|>L_{A} / 2$. In this case, the wave front first enters region $A$ after having traveled a distance $x_{1}-L_{A} / 2$, then travels inside $A$ (length $L_{A}$ ), and finally escapes region $A$ after having traveled a length $x_{1}+L_{A} / 2$. At the beginning, particles enter region $A$, and we expect an increase in $D_{\psi_{1}, \psi_{2}}(t)$. Assuming that the front moves at constant speed $v \sim 2 / \sigma$ (which can be read off from $\sigma_{t}$ at large times) the time scales after which we expect to see Markovian behavior is given roughly by $\tau_{M} \simeq \sigma\left(x_{1}+L_{A} / 2\right) / 2$. In general, the appearance of a region of non-Markovianity can be observed as long as the initial wave functions are sufficiently localized, so that the initial front does not surpass region $A$, i.e. roughly $\sigma<\left(x_{1}+L_{A} / 2\right) / 2$.

These predictions are confirmed by our numerical experiments shown in Fig. 2. The purely Markovian behavior observed for $\left|x_{i}\right| \leq L_{A} / 2$ indicates, as it is reasonable to expect, that for infinite spatial extent, wave packets always leak out of region $A$ and never come back. An important ingredient in reaching this conclusion is the fact that, for the case considered, the spectrum is purely continuous. The presence of bound states in the spectrum may lead to oscillations of information back and forth from region $A$. This in turn may lead to a breaking of Markovianity in case such bound states are initially populated.

\section{B. Double delta barrier}

In this section we modify the free dynamical evolution by introducing two delta barriers at the boundaries of region $A$, at positions $\pm L_{A} / 2$, i.e. we consider the potential $V(x)=V_{1} \delta\left(x-L_{A} / 2\right)+V_{2} \delta\left(x+L_{A} / 2\right)$, where $V_{i}$ measures the strengths of the barriers. Eigenstates and eigenvalues of this system can be found by integrating Schrödinger's equation in the neighborhood of the barriers, and imposing continuity of the wave function. As a result one obtains a transcendental equation for the quantum number $k$, which is solved
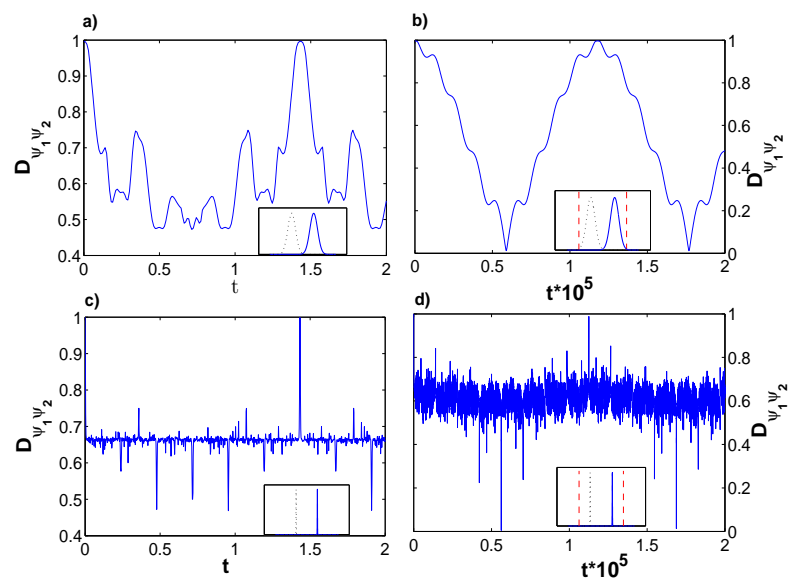

Figure 3: (Color online) Time evolution of the HS distance $D_{\psi_{1}, \psi_{2}}(t)$ between two Gaussian wave packets for various initial conditions with and without system-bath delta barriers. The insets show initial conditions and whether or not barriers are included. Note that the time scale to return to a state of large HS distance, $\tau_{r e c}=\min _{t>\tau_{d e c}}\left(\left\{t: D_{\psi_{1} \psi_{2}}(t)>1-\epsilon\right\}\right)$ is very different for the left and right columns. Also, the time scale for reconstructions $\tau_{\text {rec }}$ does not depend on the wave packet width, but the initial decay time, $\tau_{d e c}=\min _{t>0}\left(\left\{t: D_{\psi_{1} \psi_{2}}(t)<\bar{D}_{\psi_{1}, \psi_{2}}\right\}\right)$ where $\bar{D}_{\psi_{1}, \psi_{2}}:=$ $\lim _{T_{\max } \rightarrow \infty} T_{\max }^{-1} \int_{0}^{T_{\max }} D_{\psi_{1}, \psi_{2}}(t) d t$ does. a) [resp. b)] broad initial wave packets with no [resp. with] barriers. c) [resp. d)] narrow initial wave packets with no [resp. with] barriers. In all of these plots $L_{A}=2$ and $L_{B}=1$ the barriers are of strength $V_{1}=10^{6}$ and $V_{2}=2.0 \times 10^{6}$. "Narrow" Gaussians have width $\sigma=0.005$ while "broad" Gaussians have width $\sigma=0.125$.

using a numerical root finder based on the bisection method [20]. Again, for finite size $L$, the spectrum is purely (countably) discrete. The initial state is expressed in the Hamiltonian eigenbasis according to $\psi_{j}(0, x)=\sum_{k} \phi_{k}(x)\left\langle\phi_{k} \mid \psi_{j}\right\rangle$, keeping as many terms in order to reach a fidelity of at least 0.99. The eigenfunctions themselves are just piece-wise con- 


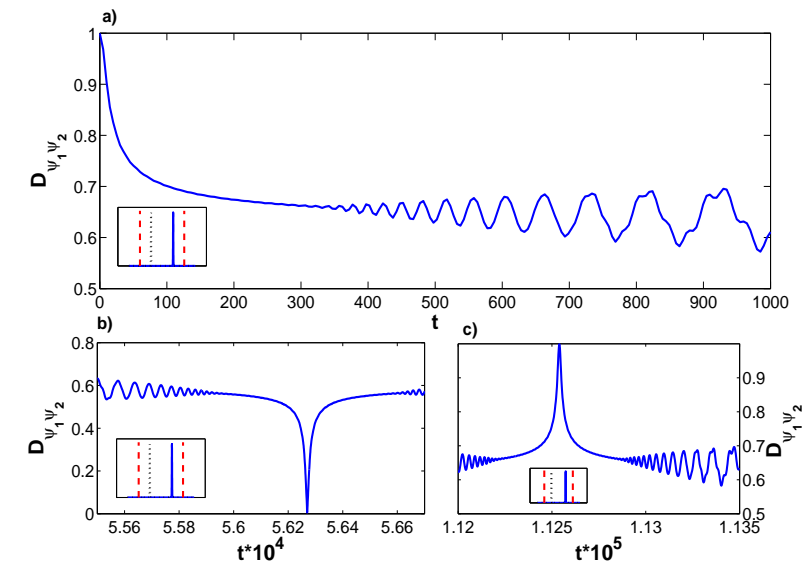

Figure 4: (Color online) Three characteristic features in the time evolution, all for narrow initial wave packets (indicated in the insets). a) Initial smooth decay of $D_{\psi_{1}, \psi_{2}}(t)$ followed by non-Markovian oscillations. b) Non-monotonic decay (and subsequent increase) c) Reconstruction consisting of a smooth increase in the HS distance back to 1 , followed by a smooth decay analogous to (a). These features reoccur at almost periodically at larger times but do eventually weaken and disappear. In all of these plots $L_{A}=2$ and $L_{B}=1$ the barriers are of strength $V_{1}=10^{6}$ and $V_{2}=2.0 \times 10^{6}$

tinuous combinations of plane waves in region $A$ and $B$. All this information is then inserted in Eq. (13) and eventually into Eq. (17).

Introducing delta barriers of strength $V_{i}$, separating system and bath, causes several profound modifications of the time evolution, as observed in Fig. 3 (b) and (d). First, the time scale for recurrences is increased by several orders of magnitude, as this is now controlled by the tunneling probabilities through the barriers. This can be defined as $\tau_{\text {rec }}=\min _{t>\tau_{\text {dec }}}\left(\left\{t: D_{\psi_{1} \psi_{2}}(t)>1-\epsilon\right\}\right)$ where $\tau_{d e c}=\min _{t>0}\left(\left\{t: D_{\psi_{1} \psi_{2}}(t)<\overline{D_{\psi_{1} \psi_{2}}}\right\}\right)$ and $\bar{D}_{\psi_{1}, \psi_{2}}:=$ $\lim _{T_{\max } \rightarrow \infty} T_{\max }^{-1} \int_{0}^{T_{\max }} D_{\psi_{1}, \psi_{2}}(t) d t$. Similarly, the characteristic time scale of the faster oscillations due to the dispersing wave packets is modified by the tunneling process through the barriers, albeit in a non-trivial manner. Although the effective number of states does not seem to be drastically modified, now a dominant mode is singled out among the $N^{*}$ states. This is clearly visible as an underlying modulation in Fig. 3 (b) and (d).

In addition to these two phenomena, one also observes an extended pseudo-Markovian initial transient decay in the HS distance, best seen Fig. 4(a), where we zoom into the earlytime response. During this transient period, the two initial wave packets become less distinguishable. The time scale $t_{N M}$ where non-Markovian oscillations set in will be discussed later.

In Fig. 4(b) we observe an event where $D_{\psi_{1}, \psi_{2}}(t)$ distance sharply decays at some time and the states become almost indistinguishable in region $A$. We also observe in Fig.4(c) that the initial wave functions become again perfectly distinguishable (i.e. orthogonal: $D_{\psi_{1}, \psi_{2}}(t)=1$ ).

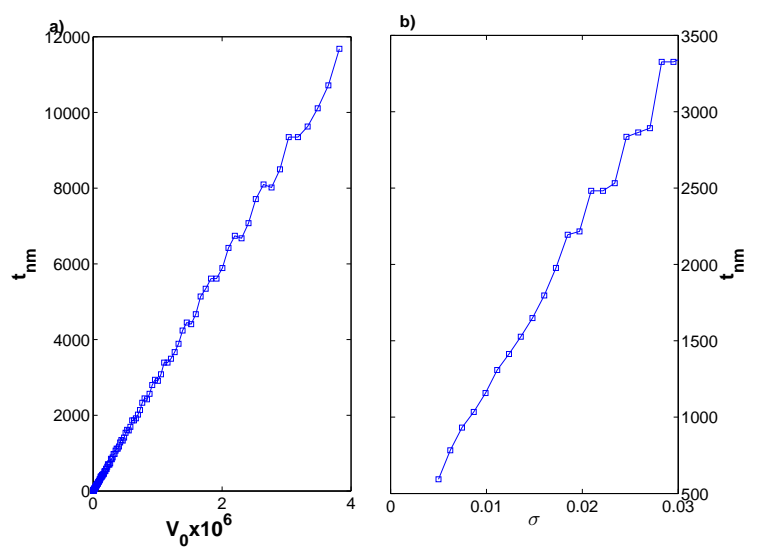

Figure 5: (Color online) Control of the timescale $t_{N M}$ for the onset of non-Markovian oscillations following the initial transient decay of the HS distance. a) $t_{N M}$ versus barrier strength $V_{0}\left(V_{1}=V_{0}\right.$ and $\left.V_{2}=2 V_{0}\right)$. b) $t_{N M}$ versus the width of the initial wave packets, $\sigma$. The dependence is approximately linear in both cases. $t_{N M}$ is operationally defined as the time when the HS distance $D_{\psi_{1}, \psi_{2}}(t)$ increases by $10 \%$ from its minimum value before $t_{N M}$. Main plots are on a log-log scale, whereas insets are on a linear scale.

The pseudo-Markovian event observed in Fig. 4 clearly defines a characteristic time scale $t_{N M}$ which is the transient for which the system behaves in a Markovian fashion before non-Markovian oscillations occur [26]. We have operationally defined $t_{N M}$ as the time when the HS distance $D_{\psi_{1}, \psi_{2}}(t)$ increases by $10 \%$ from its minimum value before $t_{N M}$. We have verified that this time constant $t_{N M}$ scales linearly with the strength of the barriers $V_{0}$, (for the case $V_{1}=V_{0}$ and $\left.V_{2}=2 V_{0}\right)$, and with the width of the initial Gaussian wave packets, $\sigma$ (see Fig. 5). Indeed, stronger barriers mean longer tunneling times, and therefore a longer time for the particles to return from the bath (Fig. 5 (a)). On the other hand narrower wave packets lead to higher occupancy of the highenergy modes which can more easily pass through the barriers (Fig.5(b)).

It can be argued that the dips and peaks observed in Figs. 4 (b) and (c) are artifacts of a very fine-tuned system-bath aspect ratio. Let us therefore examine more generic situations with variable bath size. This will also allow us to explore the level of control we can exert on the system by tuning feedback effects due to variable bath size, as well as examine the crossover to a more conventional notion of bath in the limit $L_{B} \rightarrow \infty$.

The fine tuning required to control the tunneling can be seen in Fig. 6 (a)-(d). Fig. 6 (a) shows the time averaged HS distance $\bar{D}_{\psi_{1}, \psi_{2}}:=\lim _{T_{\max } \rightarrow \infty} T_{\max }^{-1} \int_{0}^{T_{\max }} D_{\psi_{1}, \psi_{2}}(t) d t$ versus size of the bath. One observes that only at very specific values of $L_{B}$ a significant deviation from perfect distinguishability is achieved. Comparison to Fig. 6(d) which shows average probability for one of the particles to be found in the system, indicates that the underlying cause of this lack of distinguishability is that the wave function cannot significantly tunnel out of the system for most values of $L_{B}$. We notice 

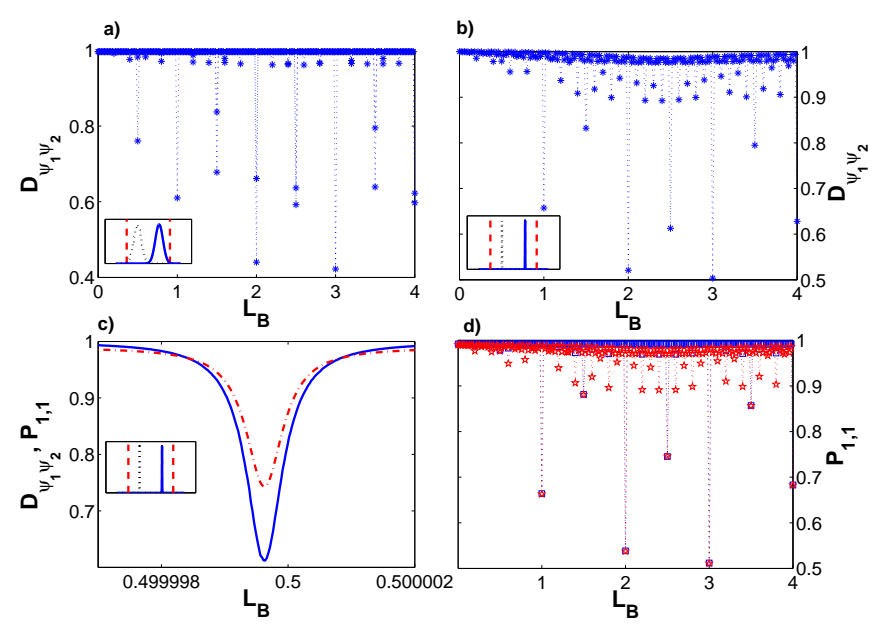

Figure 6: (Color online) Time averaged quantities as a function of bath size $L_{B}$ for fixed system size $L_{A}=2$. a) $\bar{D}_{\psi_{1}, \psi_{2}}$ versus $L_{B}$ for broad initial Gaussians: narrow dips in trace distance occur at lengths for which a significant portion of modes are able to tunnel into the bath. b) Same as (a), but with initially narrow Gaussians. c) A zoom on the peak seen in (b) at $L_{B}=1$. Note that $\bar{D}_{\psi_{1}, \psi_{2}}$ increases smoothly as $L_{B}$ is moved away from $1 / 2$. This plot also shows the time average of $p_{1,1}$ as a (red) dashed line. d) Time average of $p_{1,1}$ versus $L_{B}$ for narrow Gaussians, (red) stars, and broad Gaussians, (blue) squares. Note the similarity between these plots and plots (a) and (b). In all of these plots barriers are of strength $V_{1}=10^{6}$ and $V_{2}=2.0 \times 10^{6}$, "narrow" Gaussians have width $\sigma=0.005$ whereas "broad" Gaussians have width $\sigma=0.125$.

that in the case of narrow initial Gaussians (Fig. 6 (b)) the particle is able to tunnel out of the system for more values of $L_{B}$, as more eigenmodes of the system have significant amplitudes, but otherwise this result is analogous to the one shown in Fig. 6(a).

The reason for the behavior seen in Fig. 6relates to the fact that the energy scale of all eigenmodes of the system considered here is much smaller than the barrier strengths. For this reason, the only modes which are allowed to have appreciable amplitudes both in the system and the bath are those where both of the barriers are very close to a node.

Fig. 6 (c) shows that both the average HS distance and the average probability to be in the bath can be controlled by fine tuning $L_{B}$. As the ratio of $L_{B}$ vs. $L$ is moved further from away from $1 / 2$, the modes become separated into those which are isolated in the system, and those which are isolated in the bath. As this happens, tunneling is reduced, and consequently the particles become more distinguishable on average.

We now turn our interest to the case where $L_{A}$ is fixed and $L_{B} \rightarrow \infty$. Here we set the two barrier strengths equal for simplicity. In this case the particles do not return after escap- ing the system, so the evolution is Markovian. Fig. 7 shows the resulting trace distance for the case of a pair of initially narrow Gaussians. As expected this plot indicates Markovian decay as the wave functions escape the system.

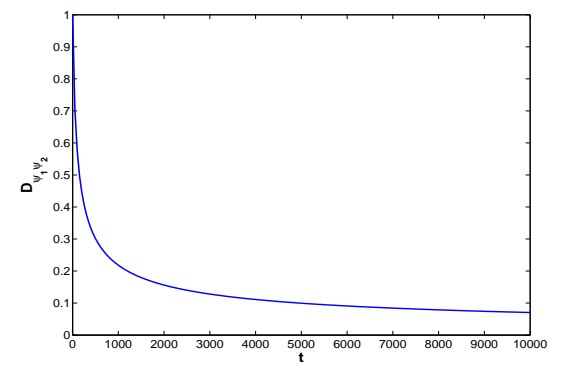

Figure 7: (Color online) Monotonic ("Markovian") decay of the HS distance versus time for a pair of initially narrow Gaussians with $L_{B} \rightarrow \infty, L_{A}=1$ and $V_{1}=V_{2}=V_{0}=10^{6}$.

\section{CONCLUSIONS}

In summary, we have investigated non-Markovian effects arising from a tunable finite bath. These can be quantified by a measure that tracks particles flow out of and into a system that is connected to the bath. We have identified various time scales for rephasing events that depend non-trivially on the bath size, on the tunneling barrier potentials between the system and the bath, and on the shape of the wave functions with which the evolution is initiated. In particular, we found that substantial rephasing can be achieved by fine tuning the bath length and by choosing initial states with significant high frequency components, allowing the wave packets to tunnel efficiently between the system and the bath. One can envision physical realizations of such a setup in the context of nanoelectronics and nanophotonics. For example, a photonic microcavity can act as the system, connected via semitransparent mirrors to an external cavity that acts as the bath. Then the transparency of the mirrors corresponds to the barrier potential, and the length of the external cavity sets the time scale for major rephasing events. An implementation in the context of nanoelectronics may be even more interesting, because in this case the effects of electron interactions on nonMarkovian system dynamics could be studied as well.

Acknowledgements: The authors would like to thank Tameem Albash and Paolo Zanardi for useful discussions. The numerical computations were carried out on the University of Southern California high performance supercomputer cluster. This research has been supported by the ARO MURI grant W911NF-11-1-0268. S. Haas would like to thank the Humboldt Foundation for support.
[1] Lorenzo Campos Venuti and Paolo Zanardi. Universality in the equilibration of quantum systems after a small quench. Phys. Rev. A, 81(3):032113, March 2010.
[2] Lorenzo Campos Venuti, Sunil Yeshwanth, and Stephan Haas. Equilibration times in clean and noisy systems. Phys. Rev. A, 87(3):032108, March 2013. 
[3] Nicholas Chancellor, Christoph Petri, and Stephan Haas. Nonmarkovian equilibration controlled by symmetry breaking. Phys. Rev. B, 87(18):184302, May 2013.

[4] G. Lindblad. On the generators of quantum dynamical semigroups. Communications in Mathematical Physics (19651997), 48(2):119-130, 1976.

[5] Heinz-Peter Breuer, Elsi-Mari Laine, and Jyrki Piilo. Measure for the degree of non-markovian behavior of quantum processes in open systems. Phys. Rev. Lett., 103(21):210401, November 2009.

[6] Tomaž Prosen. Third quantization: a general method to solve master equations for quadratic open fermi systems. New J. Phys., 10(4):043026, April 2008.

[7] Giuliano Benenti, Antonio D'Arrigo, and Giuseppe Falci. Enhancement of transmission rates in quantum memory channels with damping. Phys. Rev. Lett., 103(2):020502, July 2009.

[8] Ángel Rivas, Susana F. Huelga, and Martin B. Plenio. Entanglement and non-markovianity of quantum evolutions. Phys. Rev. Lett., 105(5):050403, July 2010.

[9] Laura Mazzola, César A. Rodríguez-Rosario, Kavan Modi, and Mauro Paternostro. Dynamical role of system-environment correlations in non-markovian dynamics. Phys. Rev. A, 86(1):010102, July 2012.

[10] Shunlong Luo, Shuangshuang Fu, and Hongting Song. Quantifying non-markovianity via correlations. Phys. Rev. A, 86(4):044101, October 2012.

[11] Bogna Bylicka, Dariusz Chruściński, and Sabrina Maniscalco. Non-markovianity as a resource for quantum technologies. arXiv e-print 1301.2585, January 2013.

[12] Dariusz Chruściński and Andrzej Kossakowski. Nonmarkovian quantum dynamics: Local versus nonlocal. Phys. Rev. Lett., 104(7):070406, February 2010.

[13] Dariusz Chruściński, Andrzej Kossakowski, and Ángel Rivas. Measures of non-markovianity: Divisibility versus backflow of information. Phys. Rev. A, 83(5):052128, May 2011.

[14] Tomaž Prosen and Thomas H. Seligman. Quantization over boson operator spaces. J. Phys. A: Math. Theor., 43(39):392004, October 2010.

[15] Tomaž Prosen. Spectral theorem for the lindblad equa- tion for quadratic open fermionic systems. J. Stat. Mech., 2010(07):P07020, July 2010.

[16] J. Eisert and T. Prosen. Noise-driven quantum criticality. arXiv:1012.5013, December 2010.

[17] John Watrous. Lecture notes on theory of quantum information. https://cs.uwaterloo.ca/ watrous/LectureNotes.html, 2011.

[18] Lorenzo Campos Venuti and Paolo Zanardi. Unitary equilibrations: Probability distribution of the loschmidt echo. Phys. Rev. A, 81(2):022113, February 2010.

[19] Juho Häppölä, Gábor B. Halász, and Alioscia Hamma. Universality and robustness of revivals in the transverse field $\mathrm{XY}$ model. Phys. Rev. A, 85(3):032114, March 2012.

[20] William T Vetterling, William H Press, Brian P Flannery, and Saul A Teukolsky. Numerical Recipes in C: The Art of Scientific Computing. Cambridge University Press, Cambridge; New York, 1992.

[21] As will be clear below, the computation simply requires diagonalization of a matrix of size $O(N)$ for a system of $N$ particles.

[22] In vector notation Eq. (3) becomes $\mid \dot{\Gamma} \gg=$ $\left[\mathbb{I} \otimes X^{T}+X^{T} \otimes \mathbb{I}\right]|\Gamma \quad \gg \quad-| Y \quad \gg . \quad$ Calling $\mathcal{M}=\left[\mathbb{I} \otimes X^{T}+X^{T} \otimes \mathbb{I}\right]$ the solution is $\mid \Gamma(t) \gg=$ $e^{t \mathcal{M}}(|\Gamma(0) \gg-| \Gamma(\infty) \gg)+\mid \Gamma(\infty) \gg$ with unique asymptotic state $\left|\Gamma(\infty) \gg=\mathcal{M}^{-1}\right| Y \gg$ in case $\mathcal{M}$ is invertible. Under the above hypothesis the spectrum of $\mathcal{M}$ has non positive real part and the result (4) follows.

[23] We are aware that a particle-number conserving evolution is not consistent with Eqns. (1) and (3). In practice we are asking if it is possible to have a Markovian evolution [of the form of Eqns. (1) and [3] for the subsystem $A$ giving rise to the observed matrix $R$ in this subsystem.

[24] The error function is defined as $\operatorname{Erf}(z):=\pi^{-1 / 2} 2 \int_{0}^{z} e^{-t^{2}} d t$.

[25] Indeed, consider $U_{L R}$ the unitary operator which implements the Left-Right inversion around the origin. Then, at all times, for symmetric potentials $V(x), U_{L R}\left|\psi_{1}(t)\right\rangle=e^{i \phi}\left|\psi_{2}(t)\right\rangle$. Then $p_{1,1}(t)=\left\langle\psi_{1}(t)\left|U_{L R}^{\dagger} U_{L R} P_{A} U_{L R}^{\dagger} U_{L R}\right| \psi_{1}(t)\right\rangle=$ $\left\langle\psi_{2}(t)\left|P_{A}\right| \psi_{2}(t)\right\rangle=p_{2,2}(t)$ since $\left[P_{A}, U_{L R}\right]=0$.

[26] Operationally $t_{N M}$ is the first occurrence of time $t$ for which $\sigma\left(t, \Gamma_{1}, \Gamma_{2}\right)>0$ 